\title{
On the abundances of GRO J1655-40^
}

\author{
C. Foellmi ${ }^{1,2}$, T. H. Dall ${ }^{3}$, and E. Depagne ${ }^{1,4}$ \\ 1 European Southern Observatory, 3107 Alonso de Cordova, Casilla 19001, Vitacura, Santiago, Chile \\ e-mail: cfoellmi@eso.org \\ 2 Laboratoire d'Astrophysique, Observatoire de Grenoble, BP 53, 38041 Grenoble Cedex 9, France \\ 3 Gemini Observatory, 670 N. A'ohoku Place, Hilo, HI 96720, USA \\ ${ }^{4}$ Departamento de Astronomía y Astrofísica, Pontificia Universidad Católica de Chile, Campus San Joaquín, Vicuña Mackenna 4860, \\ Casilla 306, Santiago 22, Chile
}

Received 16 January 2007 / Accepted 1 February 2007

\begin{abstract}
Context. The detection of overabundances of $\alpha$-elements and lithium in the secondary star of a black-hole binary provides important insights about the formation of a stellar-mass black-hole. $\alpha$-enhancement might theoretically also be the result of pollution by the nucleosynthesis occurring during an outburst, or through spallation by the jet.

Aims. We study the abundances, and their possible variations with time, in the secondary star of the runaway black-hole binary GRO J1655-40, in order to understand their origin.

Methods. We present a detailed comparison between a Keck spectrum obtained in 1998 found in the literature, archival VLT-UVES data taken in 2004 and new VLT-UVES spectra obtained early 2006. We carefully determine the equivalent widths of different $\alpha$-elements (Mg, O, Ti, S and Si) with their associated uncertainty. We use the well-studied comparison star HD 156098 as well as synthetic spectra to match the spectrum of GRO J1655-40 in order to determine the abundances of these elements.

Results. We see no significant variations of equivalent widths with time. Our fit using HD 156098 reveals that there is significant overabundance of oxygen in all our spectra, but no overabundances of any of the other $\alpha$-elements. Finally, we do not detect the lithium line at $6707 \AA$.

Conclusions. We show that there is no detected pollution in GRO J1655-40 after the burst in 2005. Moreover, we argue that uncertainties in the equivalent widths were previously underestimated by a factor of $\sim 3$. Consequently, our results challenge the existence of general overabundances of $\alpha$-elements observed in this galactic black-hole binary, and thus the accepted interpretation that they are of supernova origin. The physical cause of the overabundance of oxygen remains unclear.
\end{abstract}

Key words. stars: abundances - X-rays: binaries - stars: binaries: spectroscopic - stars: individual: GRO J1655-40

\section{Introduction}

In 1999, Israelian and coworkers published evidences of overabundances of $\alpha$-elements by a factor 6 to 10 compared to solar, observed in the secondary star of the runaway black-hole GRO J1655-40 (Israelian et al. 1999), discovered in 1994 by BATSE (Zhang et al. 1994). The spectrum has been taken when GRO J1655-40 was in quiescence, on May 24, 1998 with the $10-\mathrm{m}$ Keck I telescope. The authors made a LTE modeling of the spectrum (corrected for non LTE effects), and overabundances were explained by a probable pollution by the original supernova, also responsible for the kick velocity of the system. This explanation has been extensively studied, for instance, by Podsiadlowski et al. (2002) who conclude that the black-hole was formed through a two-steps black-hole formation scenario, with substantial fallback.

Recently, Foellmi et al. (2006, hereafter Paper I) have presented an analysis of archival VLT-UVES spectra of GRO J1655-40 obtained in 2004, when the system was in

* Based on data obtained at the Very Large Telescope, European Southern Observatory, under program IDs 276.D-5027(A) and 073.D0473(A). quiescence. Paper I is dedicated to the problem of the distance of GRO J1655-40, but we found during the analysis of the spectrum that overabundances of $\alpha$-elements (in particular $\mathrm{Mg}$ ) were not detected, casting doubt on the overabundances in the Keck spectrum. If the disappearance of such overabundances between 1998 and 2004 was real, it was certainly associated also with a short timescale phenomenon. A mechanism of flare-pollution is known to occur in Cataclysmic Variables (Stehle \& Ritter 1999), but seems to have never been observed in microquasars. Models invoke accretion disk nucleosynthesis (e.g. Mukhopadhyay \& Chakrabarti 2000) that might produce in particular large amounts of lithium (e.g. Martin et al. 1994; Guessoum \& Kazanas 1999), or misalignment of the jet with the accretion disk spin (e.g. Butt et al. 2003).

GRO J1655-40 is an ideal laboratory for studying these questions. Before the Keck observation in 1998, the source has been in outburst the year of its discovery (1994, see reference above), in 1995 and 1996 (see Orosz et al. 1997, for a chronology of the activity of GRO J1655-40 from 1994 to 1997). Interestingly, GRO J1655-40 has been in quiescence between 1999 and early 2005 where a new outburst occurred (see e.g. Brocksopp et al. 2006). It has reached again its complete 
quiescent level in October 2005 (ATel \#644'). Moreover, GRO J1655-40 is considered in the literature as a misaligned microquasar (e.g. Maccarone 2002) since the inclination angle of the system is around $70^{\circ}$ (Greene et al. 2001), while the jet angle is at $85^{\circ}$ according to Hjellming \& Rupen (1995). However, in Paper I we have shown that the distance of GRO J1655-40 is much smaller than previously used values. Thus, adopting a distance of $1.0 \mathrm{kpc}$, as we inferred in Paper I, the radio data of Hjellming \& Rupen (1995) imply a jet angle of $72^{\circ} \pm 2$. In this case, GRO J1655-40 is not misaligned.

We have conducted a new VLT-UVES program at the beginning of 2006. We present in this Letter the comparison between the results of Israelian et al. (1999), and the UVES spectra taken in 2004 and 2006, to check the possible presence of lithium, and to examine whether overabundances of $\alpha$-elements in the secondary star of GRO J1655-40 exist, and whether they are variable.

\section{Observations}

VLT-UVES spectra taken in 2004 are described in Paper I. We obtained new spectra of GRO J1655-40 with VLT-UVES on the nights Feb. 18, March 7, 8 and 19, 2006. In total, five spectra with a central wavelength of $5800 \AA$ were acquired, covering the exact same spectral domain as the 2004 spectra: from 4785 to $5755 \AA$ and 5835 to $6805 \AA$. One spectrum was also obtained with a central wavelength of $8600 \AA$, covering the regions 6680 to $8540 \AA$ and 8650 to $10080 \AA$, matching the wavelength range presented by Israelian et al. (1999). The resolving power of the spectra is 45000 . Each individual spectrum has a $S / N$ of about 40 to 60 in the continuum. They have been reduced the same way as described in Paper I, and shifted to the heliocentric restframe. In order to achieve a higher Signal-to-Noise ratio, we have crosscorrelated, shifted and combined the spectra together. The resulting spectra have a $S / N$ between 100 and 150 in the continuum.

\section{Analysis}

As confirmed in Paper I, the secondary star in GRO J1655-40 is a F6IV star with plenty of absorption lines, and a rotational velocity of $94 \mathrm{~km} \mathrm{~s}^{-1}$. As an immediate consequence it is extremely hard to find line-free regions in the spectrum, and thus to safely determine the continuum level.

\subsection{Temporal variations of equivalent widths}

In order to carefully measure the equivalent widths $(E W \mathrm{~s})$, we have: (1) fit the continuum of the whole spectrum with a loworder polynomial choosing small line-free regions of the spectrum; (2) cross-correlate and shift the 2006 spectra to match the 2004 velocity; (3) linearly rebin the 2006 spectra to the exact same wavelength dispersion of the 2004 spectra; (4) define com$m o n$ wavelength limits to the line wings to measure the $E W \mathrm{~s}$. The $E W$ s are summarized in Table 1, where the values of Israelian et al. (1999) are also reproduced. We have computed the internal uncertainties of the $E W \mathrm{~s}$ using the recent work by Vollmann \& Eversberg (2006). We also tested our EW measurements with gaussian convolution and binning before the continuum fit. It usually gives smaller values of $E W \mathrm{~s}$, since pre-continuum fit operations tends to smear out the lines with the continuum, thus lowering the contrast with the lines.

\footnotetext{
${ }^{1}$ See http://www . astronomerstelegram.org/
}

Table 1. Equivalent widths ( $E W \mathrm{~s}$, in units of $\mathrm{m} \AA$ ) of various absorption lines observed in 1999, 2004 and 2006. The values of 1999 are taken unchanged from Israelian et al. (1999). A ${ }^{+}$sign indicates values actually quoted by the authors from the work by Shahbaz et al. (1999). Horizontal lines delimitate groups of lines for which only one $E W$ can be measured.

\begin{tabular}{|c|c|c|c|c|}
\hline$\overline{\text { Line }}$ & Ion & $\begin{array}{l}1999 \\
(\mathrm{~m} \AA)\end{array}$ & $\begin{array}{l}2004 \\
(\mathrm{~m} \AA)\end{array}$ & $\begin{array}{l}2006 \\
(\mathrm{~m} \AA)\end{array}$ \\
\hline 5167.32 & Mg I & - & $940 \pm 70$ & $890 \pm 65$ \\
\hline 5172.68 & Mg I & - & $420 \pm 50$ & $475 \pm 45$ \\
\hline 5183.60 & $\mathrm{Mg} \mathrm{I}$ & - & $510 \pm 50$ & $505 \pm 50$ \\
\hline 6633.42 & $\mathrm{Fe} \mathrm{I}$ & & & \\
\hline 6633.75 & $\mathrm{Fe} \mathrm{I}$ & $105 \pm 15$ & $\$ 100$ & $\sim 100$ \\
\hline 6634.12 & $\mathrm{Fe} \mathrm{I}$ & & & \\
\hline 6663.24 & Fe I & $80 \pm 15^{+}$ & $\sim 100$ & $\sim 100$ \\
\hline 6663.44 & $\mathrm{Fe} \mathrm{I}$ & & & \\
\hline 6677.99 & $\mathrm{Fe} \mathrm{I}$ & $130 \pm 15^{+}$ & $\gtrsim 100$ & $\$ 100$ \\
\hline 6749.03 & $\mathrm{Cr} I$ & $80 \pm 15^{+}$ & $\sim 100$ & $180 \pm 60$ \\
\hline 6750.16 & $\mathrm{Fe} \mathrm{I}$ & & & \\
\hline 7771.95 & $\mathrm{O} I$ & & & \\
\hline 7774.17 & O I & $1050 \pm 80$ & & $1190 \pm 100$ \\
\hline 7775.39 & O I & & & \\
\hline 7780.56 & Fe I & $90 \pm 15$ & & $160 \pm 30$ \\
\hline 8446.24 & O I & & & \\
\hline 8446.35 & O I & $530 \pm 50$ & & $460 \pm 60$ \\
\hline 8446.75 & O I & & & \\
\hline 8467.15 & Ti I & & & \\
\hline 8468.40 & $\mathrm{Fe} \mathrm{I}$ & & & $560 \pm 110$ \\
\hline 8468.50 & Ti I & & & \\
\hline 8693.93 & S I & $230 \pm 25$ & & $\sim 100$ \\
\hline 8694.62 & S I & & & \\
\hline 8728.01 & Si I & $250 \pm 25$ & & $\gtrsim 100$ \\
\hline 8728.59 & Si I & & & \\
\hline 8736.02 & $\mathrm{Mg}$ I & $200 \pm 20$ & & $150 \pm 60$ \\
\hline 8736.03 & $\mathrm{Mg} \mathrm{I}$ & & & \\
\hline
\end{tabular}

Our tests show clearly that the continuum level is the main source of uncertainty, rather than the quality of the spectra themselves. Given the $S / N$ ratio being around 100 or slightly above, we find impossible to define the continuum level to better than $1 \%$, especially given the large number of absorption lines, and the rotational broadening often causing blends. This is a crucial point, since none of the lines in Table 1 are actually isolated lines. We have thus recomputed the EWs with identical wavelength limits but artificial vertical shifts of \pm 0.01 continuum units, and square added the uncertainties to the previous ones. This contribution to the uncertainty largely dominates, by 60 to $95 \%$.

Moreover, it appeared meaningless to measure $E W$ s smaller or close to $100 \mathrm{~m} \AA$, even if we rebin the spectra to a resolution of 5000 to increase $S / N$ ratio. For instance, the blend of iron lines at $\sim 6633 \AA$ can have an $E W$ between 0 and $200 \mathrm{~m} \AA$ depending on the order of the fitting polynomial. Thus, our uncertainties are systematically larger by a factor of $\sim 3$ compared to that of Israelian et al. (1999), although our spectra are of better quality ( $S / N \sim 100-150$ vs. 35$)$ and a slightly higher resolving power (45000 vs. $\sim 30000$, assuming a 2.5 pix resolution element for the Keck spectrum²).

Given the uncertainties, it can be seen that there is no obvious $E W$ variations between our UVES spectra in 2004 and 2006. The comparison between 1998 and 2004 shows no

\footnotetext{
${ }^{2}$ Some of the overabundances reported in Israelian et al. (1999) were obtained with an even lower-resolution spectrum by Shahbaz et al. (1999).
} 


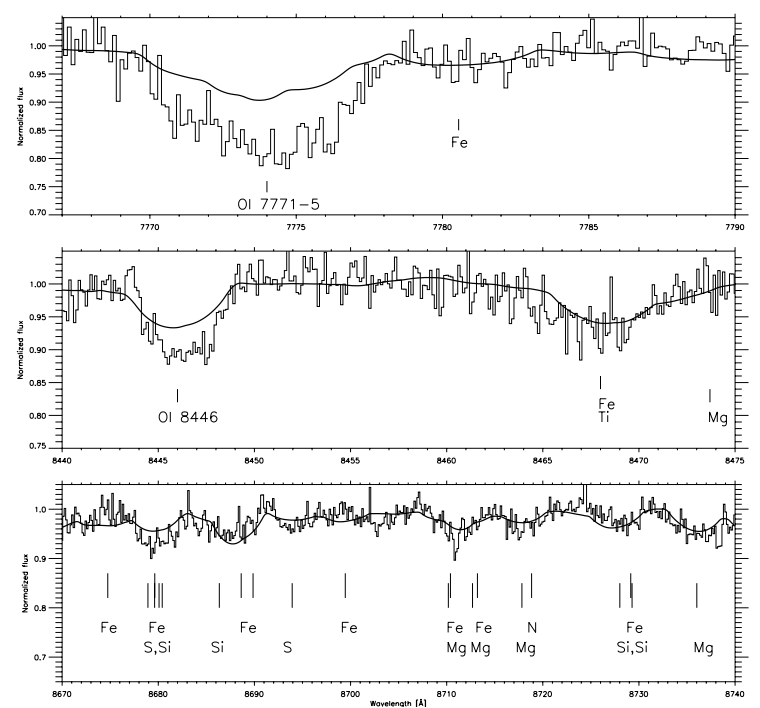

Fig. 1. UVES 2006 spectrum of the oxygen triplet $\lambda 7771-5$ (top), the O I $\lambda 8446$ line (middle), and the region around $8700 \AA$, containing many lines of the $\alpha$-elements. We have indicated the major constituents of the line blends, plotting the $\alpha$-element indicators and identifications below those of the other elements. The overabundance of oxygen is obvious, while the abundances of the other $\alpha$-elements are comparable with the abundances in HD 156098 (solid line). These three regions correspond almost exactly to the plots of the 1998 Keck-HIRES spectrum (Israelian et al. 1999, Fig. 1), with a few modifications due to UVES inter-order gaps.

systematic significant variations neither. Thus, the overall metallicity and the $\alpha$-element abundances of GRO J1655-40 have remained constant since 1998. Moreover, we do not detect the lithium $\lambda 6707$ line at a level above our uncertainties in any of our spectra.

\section{2. $\alpha$-element abundances}

In order to study the abundances of the secondary star in GRO J1655-40, we performed both a LTE spectral synthesis of selected regions using MOOG (Sneden 1973), and spectral comparison with artificially broadened stellar templates, thereby bypassing problems with NLTE effects which affect in particular some oxygen lines. For the templates we used the UVES POP database of spectra (Bagnulo et al. 2003) ${ }^{3}$, taking advantage of the fact that this provides spectra taken with the same instrument and setup. As explained in Paper I, and as confirmed by Israelian et al. (1999), any of the template stars explored in Table 1 of Paper I can be used without introducing noticeable uncertainties in the derived abundances. However, for consistency with Paper I, we use exclusively the slowly rotating star HD 156098 as a template (F6IV, $V=5.537 \mathrm{mag}, T_{\text {eff }}=6480 \mathrm{~K}, \log g=3.94$, $\mathrm{Fe} / \mathrm{H}=0.09$; Edvardsson et al. 1993), which corresponds almost exactly to the model used by Israelian et al. (1999). Bensby et al. (2005) confirmed the metallicity of HD 156098 and found a slight enhancement of the $\alpha$-elements $([\alpha / \mathrm{Fe}]=0.10$; average of $\mathrm{Mg}, \mathrm{Si}, \mathrm{Ca}, \mathrm{Ti}-[\mathrm{O} / \mathrm{H}] \sim 0.0-0.1)$. The synthesis and spectral subtraction was done using STARMOD (Barden 1985; Montes et al. 1995, 2000). Given the large $\alpha$-enhancement of GRO J1655-40 ([ $\alpha / \mathrm{Fe}] \sim 1.0)$ found by Israelian et al. (1999) we would expect to see clear enhancements of lines of the $\alpha$-elements with respect to the template spectrum.

\footnotetext{
${ }^{3}$ http://www.Sc.eso.org/santiago/uvespop/
}

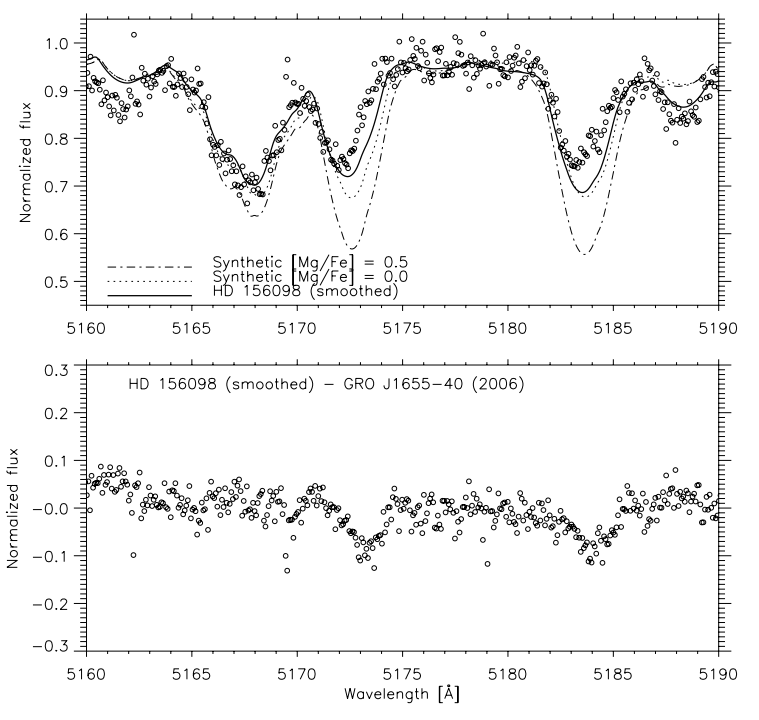

Fig. 2. One of the single UVES 2006 spectrum of GRO J1655-40 (symbols) with the F6IV broadened stellar template of HD 156098 (solid line). Also plotted are two model spectra with $[\mathrm{Mg} / \mathrm{Fe}]=0.0$ (dotted line) and $[\mathrm{Mg} / \mathrm{Fe}]=0.5$ (dot-dashed line). The apparent wavelength shift is caused by the blending of several lines with the magnesium ones. It has however no influence on $\mathrm{Mg}$ abundances. Below are plotted the residuals after subtracting the HD 156098 template spectrum from the GRO J1655-40 spectrum.

In Fig. 1 we show the general good match of comparing the spectra of GRO J1655-40 with the broadened template spectrum, using the same spectral regions as in Israelian et al. (1999). We confirm the large overabundance of oxygen, which is obvious from both sets of oxygen lines. However, we do not see any evidence for general $\alpha$-enhancement: the region around $8700 \AA$ seem to match the template very well, meaning that the abundances of $\mathrm{Ca}, \mathrm{Mg}, \mathrm{Si}, \mathrm{Ti}$, and $\mathrm{S}$ are at most only slightly overabundant, corresponding to the $[\alpha / \mathrm{Fe}]=0.10$ found in HD 156098.

To check this result, we investigated two other spectral regions around the $\mathrm{MgI}$ and $\mathrm{SI}$ triplets. For the $\mathrm{Mg}$ I triplet $\lambda \lambda 5167,5172,5183$ we also calculated synthetic spectra for a range of magnesium abundances with the same parameters as those of HD 156098. The result is shown in Fig. 2, which again reveals no obvious $\alpha$-enhancement, as the lines match the HD 156098 template very well. The Mg I triplet is known to exhibit NLTE effects, so one must be cautious when interpreting the synthetic spectra. Nevertheless, the $[\mathrm{Mg} / \mathrm{Fe}]=$ 0.0 model seem to fit HD 156098 quite well, while the $[\mathrm{Mg} / \mathrm{Fe}]=$ +0.5 model is clearly a bad fit to either star. Finally, the relative strength of the three lines can be used as a rough indicator of abundance, with the blue-most line being weaker than the other two for overabundances larger than $[\mathrm{Mg} / \mathrm{Fe}] \sim 0.3$. Changing $T_{\text {eff }}$ and $\log g$ of the model has little effect on the abundances.

The far red region of the strong S I $\lambda \lambda$ 9212,9228,9237 triplet coincides with a broad-winged hydrogen absorption line. When normalizing the spectrum of GRO J1655-40 we naturally had to exclude the area contaminated by the hydrogen line, which introduces some ambiguity in the solution. Using two equally satisfactory continuum normalizations, it is clear from Fig. 3 that sulphur is not overly abundant. 

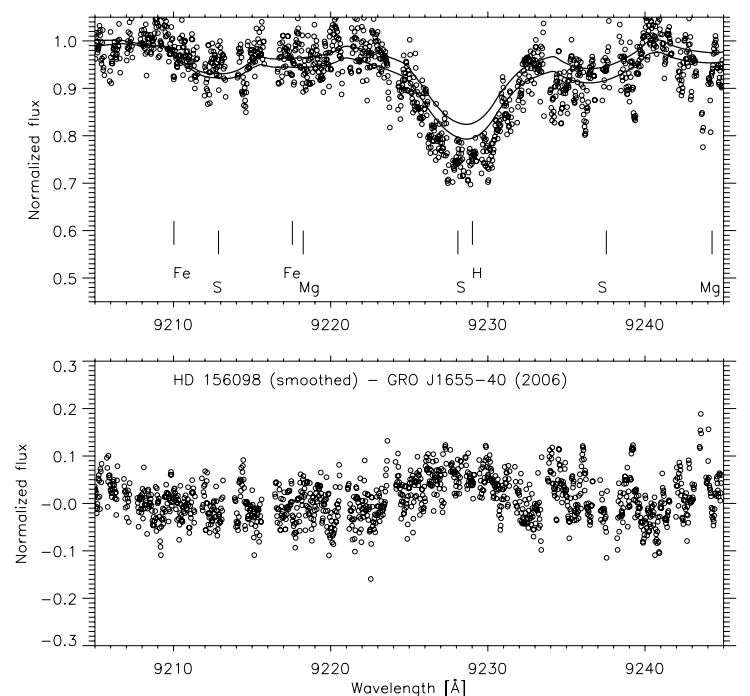

Fig. 3. UVES 2006 spectrum of GRO J1655-40 (symbols) with the F6IV broadened stellar template of HD 156098 (lower continuous line) in the region of the S I triplet. The upper continuous line show the same stellar template, but on which the difference between two acceptable normalizations of the GRO J1655-40 spectrum has been applied. It shows the amplitude of uncertainties in the continuum level in this region, and that in both cases HD 156098 is a providing a good match. Below are plotted the residuals after subtracting the HD 156098 spectrum from the best-fitting GRO J1655-40 spectrum.

\section{Discussion and conclusions}

We are led to conclude that the $\alpha$-element overabundances found by Israelian et al. (1999) were overestimated, except for oxygen, which we confirm is clearly overabundant. The physical cause of this overabundance in the secondary star of GRO J1655-40 remains unclear.

Acknowledgements. We thank the ESO Director General Dr. C. Cesarsky for accepting our DDT run early 2006. T.H.D. thanks Garik Israelian and Rafael Rebolo for useful discussions and hospitality. We thank I. F. Mirabel for useful advices and support, and the anonymous referee for useful comments.

\section{References}

Bagnulo, S., Jehin, E., Ledoux, C., et al. 2003, The Messenger, 114, 10 Barden, S. C. 1985, ApJ, 295, 162

Bensby, T., Feltzing, S., Lundström, I., \& Ilyin, I. 2005, A\&A, 433, 185

Brocksopp, C., McGowan, K. E., Krimm, H., et al. 2006, MNRAS, 365, 1203

Butt, Y. M., Maccarone, T. J., \& Prantzos, N. 2003, ApJ, 587, 748

Edvardsson, B., Andersen, J., Gustafsson, B., et al. 1993, A\&A, 275, 101

Foellmi, C., Depagne, E., Dall, T. H., \& Mirabel, I. F. 2006, A\&A, 457, 249

Greene, J., Bailyn, C. D., \& Orosz, J. A. 2001, ApJ, 554, 1290

Guessoum, N., \& Kazanas, D. 1999, ApJ, 512, 332

Hjellming, R. M., \& Rupen, M. P. 1995, Nature, 375, 464

Israelian, G., Rebolo, R., Basri, G., Casares, J., \& Martín, E. L. 1999, Nature, 401, 142

Maccarone, T. J. 2002, MNRAS, 336, 1371

Martin, E. L., Rebolo, R., Casares, J., \& Charles, P. A. 1994, ApJ, 435, 791

Montes, D., de Castro, E., Fernandez-Figueroa, M. J., \& Cornide, M. 1995, A\&AS, 114, 287

Montes, D., Fernández-Figueroa, M. J., De Castro, E., et al. 2000, A\&AS, 146, 103

Mukhopadhyay, B., \& Chakrabarti, S. K. 2000, A\&A, 353, 1029

Orosz, J. A., Remillard, R. A., Bailyn, C. D., \& McClintock, J. E. 1997, ApJ, 478, L83

Podsiadlowski, P., Nomoto, K., Maeda, K., et al. 2002, ApJ, 567, 491

Shahbaz, T., van der Hooft, F., Casares, J., Charles, P. A., \& van Paradijs, J. 1999, MNRAS, 306, 89

Sneden, C. 1973, ApJ, 184, 839

Stehle, R., \& Ritter, H. 1999, MNRAS, 309, 245

Vollmann, K., \& Eversberg, T. 2006, Astron. Nachr., 327, 862

Zhang, S. N., Wilson, C. A., Harmon, B. A., et al. 1994, IAU Circ., 6046, 1 